\title{
r.m. \\ CRISPR-Cas Systems for Diagnosing Infectious Diseases
}

\author{
Anastasiya Kostyusheva ${ }^{1}$, Sergey Brezgin ${ }^{1,2}$, Yurii Babin ${ }^{1}$, Irina Vasil'eva ${ }^{1}$, Dmitry Kostyushev ${ }^{1}$ \\ and Vladimir Chulanov ${ }^{1,3}$ \\ ${ }^{1}$ National Medical Research Center of Tuberculosis and Infectious Diseases, Ministry of Health, Moscow, \\ Russia \\ ${ }^{2}$ Institute of Immunology, Moscow, Russia \\ ${ }^{3}$ Sechenov University, Moscow, Russia \\ * Correspondence:orresponding author: Anastasiya Kostyusheva \\ Laboratory of Genetic Technologies and Translational Medicine \\ Dostoevskaya st., 4 \\ National Medical Research Center for Tuberculosis and Infectious Diseases \\ Moscow, Russia \\ +7 (925) 310-91-24 (Tel.) \\ kostyusheva_ap@mail.ru
}

\begin{abstract}
Infectious diseases are a global health problem affecting billions of people. Developing rapid and sensitive diagnostic tools is key for successful patient management and curbing disease spread. Currently available diagnostics are very specific and sensitive but time-consuming and require expensive laboratory settings and well-trained personnel; thus, they are not available in resource-limited areas, for the purposes of large-scale screenings and in case of outbreaks and epidemics. Developing new, rapid, and affordable point-of-care diagnostic assays is urgently needed. This review focuses on CRISPR-based technologies and their perspectives to become platforms for point-of-care nucleic acid detection methods and as deployable diagnostic platforms that could help to identify and curb outbreaks and emerging epidemics. We describe the mechanisms and function of different classes and types of CRISPR-Cas systems, including pros and cons for developing molecular diagnostic tests and applications of each type to detect a wide range of infectious agents. Many Cas proteins (Cas9, Cas12, Cas13, Cas14) have been leveraged to create highly accurate and sensitive diagnostic tools combined with technologies of signal amplification and fluorescent, potentiometric, colorimetric, or lateral flow assay detection. In particular, the most advanced platforms -- SHERLOCK/v2, DETECTR, or CRISPR-Chip -- enable detection of attomolar amounts of pathogenic nucleic acids with specificity comparable to that of PCR but with minimal technical settings. Further developing CRISPR-based diagnostic tools promises to dramatically transform molecular diagnostics, making them easily affordable and accessible virtually anywhere in the world. The burden of socially significant diseases, frequent outbreaks, recent epidemics (MERS, SARS and the ongoing coronoviral nCov-2019 infection) urgently need the developing of express-diagnostic tools. Recently devised CRISPR-technologies represent the unprecedented opportunity to reshape epidemiological surveillance and molecular diagnostics.
\end{abstract}

Keywords: molecular diagnostics; molecular epidemiology; HIV; HBV; HCV; HPV; Zika; Dengue; tuberculosis; SARS; MERS; nCov-2019

\section{Introduction}

Infections are one of the most daunting threats to humanity, responsible for an immense burden of disabilities and deaths ${ }^{1}$. Pandemics of influenza (Spanish flu, swine flu, bird flu), recent outbreaks of Ebola and Zika virus, deadly and wide-spread epidemics of MERS and SARS, as well as the 
ongoing outbreak of nCov-2019, originated in China in 2019, sweep across continents and emerge as the most recent examples of widespread infections reported in this century ${ }^{2-6}$.

Many infections can become chronic, often persisting through the infected person's lifetime. Chronic infections, like chronic viral hepatitis, human immunodeficiency virus (HIV) infection, and tuberculosis, are widely distributed and admittedly are the most prolific infectious disease killers. These characteristics define the exceptional significance of chronic infections for the global health ${ }^{7,8}$.

Detecting the etiologic pathogens of infectious diseases is necessary for timely treatment, risk reduction for patients and caregivers, and prevention of further spread of the pathogen, especially for the emerging and re-emerging viral infections. One conventional method used for diagnosing infectious diseases is direct isolation of pathogen nucleic acids from biological samples and their detection by polymerase chain reaction (PCR) ${ }^{9}$.Using sequential doubling, PCR exponentially amplifies target templates, providing an opportunity to detect even single copies of pathogenic genomes. Due to its high accuracy and specificity, PCR is used in many biological and medical applications, including diagnosing virtually any human pathogen. However, PCR requires qualified personnel and expensive, highly sophisticated equipment, and lacks standardized protocols, limiting its implementation in medical care settings. Importantly, PCR-diagnostics is time-consuming and cannot be used for rapid screening of large cohorts of people. The most recent nCov-2019 viral infection was shown to be frequently asymptomatic or without any immediately discernible clinical symptoms, e.g. hyperthermia ${ }^{10}$. Thus, screening of people in crowded places (planes, hospitals etc.) and timely isolation of infected persons cannot be performed with the use of infrared thermography. CRISPR-diagnostics can provide rapid, express-testing of persons-at-risk.

On the other hand, mass population screening, rapidly controlling biological hazards, preventing infection spread, and diagnosing infectious diseases in remote areas are difficult to do with PCR and remain a big challenge for public health services worldwide.

According to WHO criteria (ASSURED CRITERIA), the ideal diagnostic assay for any pathogen should be cheap and accurate, provide rapid results, be applicable in point-of-care practice, and require little or no specialized equipment and technical assistance ${ }^{11,12}$. To date, no assay fits all these requirements. Thus, inventing new, more effective methods of molecular diagnosis is urgently needed.

Discovery of the clustered regularly interspaced short palindromic repeats (CRISPR) and CRISPR-associated protein (Cas) (CRISPR-Cas) revolutionized biology and is already pushing health care systems to the era of precise molecular medicine. Using genetic engineering, CRISPR-Cas systems have been adapted for use in humans and are now being modified and enhanced at an extraordinary pace, enabling precise editing of virtually any DNA or RNA molecule in the body. CRISPR-Cas-based approaches are being tested to treat hereditary, infectious, and other diseases, as well as in methods of molecular visualization and other applications ${ }^{13,14}$.

In 2016, CRISPR-Cas systems were first utilized to detect nucleic acids for molecular diagnostics ${ }^{15}$. Until then, a number of successful CRISPR-Cas-based approaches to detect and diagnose infectious and non-infectious diseases had been invented. The revolution of CRISPR-Cas editing may spread further into the area of molecular diagnostics and replace PCR in many applications. CRISPR-Cas diagnostic tools are characterized by sensitivity and specificity comparable to those of traditional PCR, but do not require sophisticated (and therefore expensive) equipment and have a very low estimated cost. Embedding CRISPR-Cas into molecular diagnostics may reshape the profile of global diagnostic and health care systems ${ }^{16}$.

In this paper, we review classes and types of CRISPR-Cas, focusing on CRISPR-Cas systems already used for molecular diagnosis, and describe their properties, functions, and perspectives to become the ideal platforms for diagnosing infectious diseases and curbing disease outbreaks.

\section{Brief nomenclature of CRISPR-Cas systems and their characteristics}

CRISPR-Cas systems were first described 30 years ago in bacterial genomes ${ }^{17}$. Unique regions of DNA, later called spacers, were shown to be separated by short palindromic repeats in bacterial genomes. Small clusters of Cas genes, encoding proteins with nucleolytic activity, were frequently 
found located next to CRISPR repeat-spacer arrays ${ }^{18}$. Many years later, these palindromic repeats and Cas genes were shown to operate as a natural adaptive immune system providing defense against viral infections in bacteria and archaea. CRISPR-Cas function relies on effector Cas proteins and guiding CRISPR RNAs (crRNAs) ${ }^{19}$. CRISPR-Cas systems have been adapted to function in human and other mammalian cells. They can bind and cleave virtually any site of the target nucleic acid. CRISPR-Cas systems are partitioned into 2 distinct classes which are briefly described below (Table 1).

\subsection{Class 1 systems}

CRISPR-Cas class 1 systems comprise 4 types: I type, III type and IV type. Class I systems are characterized by multiple effector proteins.

CRISPR-Cas type1 systems share the effector module Cascade, composed of Cas proteins in a complex with a crRNA molecule ${ }^{20}$. The Cascade complex recognizes protospacer adjacent motif (PAM) sequences and unwinds target DNA, thus enabling crRNA to interact with the complementary DNA strand. Recognition of the target site entails recruitment of Cas3 protein which cuts the DNA strand not bound by Cascade complex ${ }^{21}$.

Functioning of CRISPR-Cas type III is based on a multi-subunit complex consisting of crRNA, Csm complexes in subtype III-A systems, and Cmr complexes in subtype III-B systems. These systems are characterized by the Cas10 protein. Cas10 has been recently shown to play a role in activating non-specific RNases Csm6 and Csx1. Target site recognition by CRISPR-Cas type III systems initiates polymerase activity of Cas10 protein followed by Cas10-mediated generation of cyclic oligo-(A)nucleotides (cOA). Binding of cOA by Csm6 activates the RNase domain of Csm6, which destroys both target RNA (many CRISPR-Cas type III systems target RNA rather than DNA molecules) and other neighboring RNA molecules ${ }^{22}, 23$

CRISPR-Cas type IV systems are commonly found in plasmids but their function remain largely unknown ${ }^{24}$.

\subsection{Class 2 systems}

Class 2 CRISPR-Cas systems include type II and the less common types V and VI, each possessing unique effector proteins ${ }^{25,26,27}$. Class 2 systems are characterized by less complex organization, as the effector module consists only of a large, multidomain, multifunctional protein.

Currently, class 2 CRISPR-Cas systems have been the ones most widely used in gene engineering due to their simplicity and highly effective gene editing. The CRISPR-Cas type II Cas9 protein targets the desired site of DNA by means of crRNA and trans-activating crRNA (tracrRNA) ${ }^{28}$.Jinek et al. created a chimeric RNA molecule (single-guide RNA or sgRNA) that combines crRNA and tracrRNA and simplifies CRISPR-Cas genome editing by reducing the 3-component system (Cas9 protein, crRNA, tracrRNA) to just 2 components (Cas9 protein and sgRNA). Cas9 protein is recruited to the target site by sgRNA and generates blunt-ended double-stranded breaks in the desired site of DNA ${ }^{29}$. Cas9 protein from Streptococcus pyogenes (SpCas9) is the most common choice in gene editing technology due to its highly effective on-target gene editing. However, frequent off-target activity of SpCas9, defined as cleavage of non-specific DNA genomic sequences similar to the target site, limits its use ${ }^{13}$. Non-specific targeting by Cas9 is based on its ability to tolerate mismatches between the sgRNA and DNA, allowing it to cut even very dissimilar sequences. More specific and clinically safe variants of CRISPR-Cas type II systems are genetically modified or evolved SpCas9 proteins (enhanced SpCas9 or eSpCas930; high-fidelity SpCas9-HF191; evoCas932, HypaCas933, sniper-Cas934) and certain orthologous Cas9 proteins from other species ${ }^{35-38}$. The latter have more restrictive PAM sequences and thus exert fewer potential off-target sites and have intrinsically lower ability to unwind DNA mismatched with the sgRNA $36,39,40$.

CRISPR-Cas type V systems include several subtypes (V-A, V-B, etc.); Cas12 proteins (Cas12a, Cas12b, etc.) are signature proteins of these systems ${ }^{41}$. Cas12a and Cas $12 b$ proteins cut the target sequences leaving sticky ends after DNA cleavage. Cas12a, also known as Cpf1, is often used in gene engineering. CRISPR-Cas type V systems require only the Cas protein and a crRNA to edit the target 
site. One of the advantages of using Cas12 instead of Cas9 for genetic engineering is its smaller size and lower tolerance of nucleotide mismatches between the target DNA and crRNA ${ }^{42}$. Sticky ends left after Cas12-mediated DNA cleavage are repaired via homologous recombination ${ }^{43}$, a type of lowerror repair, thus increasing accuracy of gene editing. More recently, Cas14 proteins, miniature Cas proteins (400-700 aa) of CRISPR-Cas type V systems, have been discovered ${ }^{44}$. Cas 14 have been shown to destroy single-stranded DNA without requiring PAM. Some Cas12 and Cas14 proteins possess socalled collateral activity: after binding target DNA, the proteins start destroying any adjacent DNA in a non-specific manner ${ }^{41}$.

CRISPR-Cas type VI systems encompass subtypes VI-A, VI-B, VI-C, and VI-D (also known as $\mathrm{C} 2 \mathrm{c} 2$ ). The signature protein of CRISPR-Cas type VI is Cas13. The unique property of these systems is the ability to recognize single-stranded RNA molecules. Type VI Cas proteins bind target RNA using a guiding crRNA (no tracrRNA), introduce a blunt-ended break, and indiscriminately degrade any adjacent single-stranded RNA ${ }^{45},{ }^{46}$.

Clearly, CRISPR-Cas systems are enormously diverse, varying in mechanisms of action, composition, and structure of their key elements ${ }^{47}$. In-depth analysis and characterization of new types and classes of CRISPR-Cas systems is on the forefront of world science, paving the way for creating new biological and diagnostic tools with the prospect to fundamentally transform the way we conceive health care systems.

Table 1. Brief summary of the key CRISPR-Cas systems used in gene editing.

\begin{tabular}{cccc}
\hline Class & Type & Effector protein & Target \\
\hline & Type I & $\begin{array}{c}\text { Multi-subunit complex } \\
\text { (signature protein Cas3) }\end{array}$ & Single-stranded DNA $^{25}$ \\
\cline { 2 - 4 } Class 1 CRISPR-Cas systems & Type III & $\begin{array}{c}\text { Multi-subunit complex } \\
\text { (signature protein Cas10) }\end{array}$ & DNA/RNA $^{25}$ \\
\cline { 2 - 4 } & Type IV & $\begin{array}{c}\text { Multi-subunit complex } \\
\text { (signature protein Csf1) }\end{array}$ & Unknown \\
\cline { 2 - 4 } Class 2 CRISPR-Cas systems & Type V & Cas9 & Double-strandedDNA ${ }^{29}$ \\
\cline { 2 - 4 } & Type VI & Cas12 & $\begin{array}{c}\text { Double-stranded DNA } \\
\text { Single-stranded DNA } \\
\text { May exhibit collateral activity 48 }\end{array}$ \\
\cline { 2 - 4 } & Cas14 & $\begin{array}{c}\text { Single-stranded DNA } \\
\text { May exhibit collateral activity }\end{array}$ \\
\hline
\end{tabular}

\section{Detection of nucleic acids by CRISPR-Cas}

A large set of different CRISPR-based methods used to detect nucleic acids has been recently described. Early technologies utilized the canonical Cas9 protein of type II CRISPR-Cas systems ${ }^{49}$ or its modified nucleolytically null, or dead, Cas9 (dCas9) protein ${ }^{50}$. A huge leap toward developing CRISPR-based molecular diagnostics was the discovery of protein collateral activity of Cas12, Cas13, and Cas14, a property that can be harnessed to amplify the specific on-target signal ${ }^{41}$. Today, many modifications and improvements have been introduced into CRISPR-based molecular platforms relying on collateral activity of CRISPR-Cas type V and type VI proteins, but the general concept remains unaltered.

\subsection{Detection of nucleic acids by CRISPR-Cas type II systems}

To date, CRISPR-Cas systems are routinely leveraged as tools for gene editing, epigenome remodeling, regulating gene transcription, and visualizing DNA/RNA sequences in living cells ${ }^{50}$. Another application of CRISPR-Cas as an instrument of molecular diagnostics proved possible only in recent years. Molecular methods for detecting nucleic acids based on CRISPR-Cas systems appear to be highly sensitive, specific, and capable of one-step detection of both RNA and DNA. Zhang et al. 
(2017) created a diagnostic test based on two dCas9 proteins fused with split domains of luciferase enzyme ${ }^{51}$. Binding of two dCas9 proteins to the adjacent target DNA results in re-constitution of luciferase and emission of luminescent signal that can be readily detected by a luminometer. As a proof of concept, this technology was shown to detect Mycobacterium tuberculosis with high specificity and sensitivity ${ }^{51}$.

In 2017, Wang et al. developed a multiplex method to detect human papillomavirus (HPV) by Cas9 protein targeting L1 and E6/E7 viral genes amplified using PCR ${ }^{52}$. This technology employs a 3step DNA detection protocol: (1) amplification of DNA using PCR; (2) nucleolytic cleavage of PCR amplicons by Cas9; and (3) amplification of cleaved fragments by PCR. The resulting PCR products are detected by gel electrophoresis or fluorescence. According to Wang and colleagues, this method increases sensitivity of the diagnostic test and helps to differentiate between HPV16 and HPV18 strains ${ }^{52}$.

Pardee and colleagues developed a technique called NASBA-CRISPR cleavage (NASBACC), which relies on the principle of toehold switch sensors and the ability of Cas9 protein to selectively cleave target DNA ${ }^{15}$. Toehold switch sensors represent programmed synthetic riboregulators able to control translation by binding trigger RNA. Riboregulators carry a hairpin structure that blocks translation in cis by sequestering the ribosome binding site and start codon. When a riboregulator is bound to complementary trigger RNA, the ribosome binding site and start codon are freed and translation is enabled. The RNA target is then amplified by NASBA so that the RNA of interest is reverse-transcribed and linked to the riboregulator. NASBA amplification system uses a battery of 3 enzymes: reverse transcriptase, RNase $\mathrm{H}$, and T7 polymerase. In the NASBA method, target RNA is converted into a cDNA-RNA hybrid using specific primers; RNase $\mathrm{H}$ then destroys the RNA in this duplex. Next, a primer is added to the reaction mixture to produce synthetic templates recognized by T7 polymerase. The results of the reaction are detected by colorimetry ${ }^{15}$. This technology effectively detects Zika and dengue viruses with the sensitivity of 1-3 fM.

Another milestone in CRISPR diagnostics was the development of CRISPR-Chip ${ }^{53}$. CRISPR-Chip combines CRISPR principles with an electronic transistor made of graphene (a single layer of carbon atoms). CRISPR-Chip uses a dCas9 protein that binds, but does not cut, target DNA. dCas9 proteins are immobilized on graphene transistors. After adding DNA isolated from biological samples, dCas 9 binds the target DNA, thereby altering the electric conductivity of graphene and the electric characteristics of the transistor. CRISPR-Chip is highly sensitive, able to detect as little as $1.7 \mathrm{fM}$ of target DNA, and the procedure is extremely rapid, taking only 15 minutes. CRISPR-Chip has been used to detect genetic mutation in clinical samples from patients with Duchenne muscular dystrophy ${ }^{53}$.

Methods of isothermal amplification are particularly important to performing molecular diagnosis in remote areas and in laboratories without specially trained personnel. CRISPR-Cas9triggered nicking endonuclease-mediated strand displacement amplification (CRISDA) method uses a combination of CRISPR-Cas9 technology and isothermal amplification methods. It uses Cas9 nickases (Cas9 proteins with a mutation in one nucleolytic domain that makes the protein able to cut a single DNA strand), and single-strand displacement amplification (SDA) of target fragments followed by detection of the signal by fluorescent peptide nucleic acid invasion-mediated endpoint measurement. Intensity of the signal can be measured by a fluorimeter. CRISDA technology helps to detect target nucleic acids with specificity of 1 nucleotide ${ }^{54}$.

Quan and colleagues described the finding low abundance sequences by hybridization (FLASH) method, developed to detect pathogens resistant to antimicrobial therapy ${ }^{55}$. FLASH uses a battery of sgRNAs together with Cas9 proteins that cut the gene of interest into small fragments suitable for further next-generation Illumina sequencing. DNA/cDNA ends are first blocked by phosphatase to prevent ligation with adapters utilized in the next step and then cut by Cas9. DNA of interest is nucleolytically cleaved resulting in fragments with non-blocked ends and thus can be linked with universal adapters, amplified by PCR, and sequenced. The authors confirmed the utility of this technology in models of pneumonia caused by gram-positive bacteria including S. aureus and of malaria-causing Plasmodium falciparum ${ }^{55}$. 
Similarly, the CRISPR-Cas9-triggered exponential amplification reaction (Cas-EXPAR) method combines the advantages of Cas9 to introduce site-specific cuts in target DNA with the isothermal EXPAR method with fluorescent signal detection ${ }^{56}$. Compared to other methods of isothermal amplification, such as NASBA, RCA, SDA, LAMP, or RPA, EXPAR is characterized by relatively high efficacy and speed of product amplification. Until now, EXPAR was not widely used in molecular diagnostics, and was applied mostly for detection of short microRNAs ${ }^{57}$. This limited utility was due to an inherent property of EXPAR that does not allow using extended DNA sequences as primers for amplification. However, CRISPR-Cas9 can be programmed to cut DNA molecules into fragments short enough to successfully implement EXPAR. Wild-type SpCas9 can target and cut only doublestranded DNA molecules, but using the so-called PAM-mers (templates of single-stranded DNA complementary to single-stranded templates with a PAM sequence) permits SpCas9 to recognize and cleave single-stranded RNA or DNA molecules. Reported sensitivity of Cas-EXPAR is $\sim 1$ amol - 10 fmol, and the limit of detection fluctuates around 0.82 amol. These values are comparable to those observed using PCR diagnostics. Due to the high specificity of Cas-EXPAR, this technology can be used to detect methylated DNA after bisulfite conversion, which changes all cytosines in DNA to uracil except for methylated cytosines (5-methylcytosine), which remain intact. Thus, a DNA site with cytosine converted to uracil during bisulfite conversion will not be recognized and cleaved by Cas 9 protein when the sgRNA targets the original (non-converted) sequence. As a result, isothermal amplification of such templates by EXPAR proceeds with low efficacy. Conversely, 5-methylcytosine remains intact during bisulfite conversion; sites with 5-methylcytosine can thus be effectively recognized and cut by Cas9, effectively amplified by EXPAR, and detected using conventional fluorescence methods.

\subsection{Detection of nucleic acids by CRISPR-Cas type V and VI}

In 2018, Doudna and colleagues presented the CRISPR-Cas diagnostic platform named DNA endonuclease-targeted CRISPR trans reporter (DETECTR ${ }^{48}$. This method relies on collateral activity of Cas12a protein activated after recognition of target RNA by Cas12a. The authors demonstrated that Cas12a protein from Lachnospiraceae bacterium ND2006(LbCas12a) exhibits non-specific collateral activity and degrades all adjacent DNA molecules after recognizing target RNA. If the reaction with Cas12a protein and targeting crRNA is complemented by single-stranded DNA-reporters (probes) and then mixed with the biological sample, crRNA-dependent recognition of pathogenic nucleic acids by Cas12a turns on collateral activity that destroys DNA probes. DNA probes are designed similarly to conventional TaqMan probes, in which one end of the reporter is bound by a fluorophore and the opposite is linked to a quencher. Degradation of the DNA probes releases fluorophores and results in stable and strong fluorescent signal detected by a fluorimeter. Additionally, DETECTR has been combined with an isothermal pre-amplification step to enrich target sequences (RPA). RPA enhances analytical sensitivity of the diagnostic test and helps to avoid the need for sophisticated and expensive equipment.

Other orthologous proteins from different organisms - AsCas12a (Acidaminococcus sp.), FnCas12a (Franciella novicida), AaCas12b (Alycyclobacillus acidoterrestris) ${ }^{41}$ - also possess collateral activity and can be used to create diagnostic platforms by the same principle as DETECTR.

DETECTR was provisionally used to detect HPV and differentiate between HPV16 and HPV18, the most pro-oncogenic types of HPV. In this setting, two crRNAs were designed to target the hypervariable V loop of the L1 gene, which differs by only 6 nucleotides betweenHPV16 and HPV18types. In cell culture, DETECTR effectively discriminated between these types of HPV. In crude DNA extracts, DETECTR identified HPV16 in 25 of 25 cases and HPV18 in 23 of 25 cases, provisionally determined by PCR. Notably, DETECTR analysis takes only $1 \mathrm{~h}$ to complete ${ }^{48}$.

Also in 2018,Doudnaet al. first characterized a highly diverse family of CRISPR-Cas systems similar to CRISPR-Cas type $\mathrm{V}^{44}$. In this system, the signature protein Cas14 PAM-independently cleaves single-stranded DNA molecules. Like Cas12 proteins, Cas14 exhibits collateral activity and cuts any adjacent DNA molecules, making Cas14 proteins useful in CRISPR-based diagnostics. Contrary to Cas12, Cas14 has lower tolerance to nucleotide mismatches between sgRNA and the 
target template; the internal seed-sequence is very sensitive to nucleotide mismatches, which greatly reduces on-target activity of Cas14. This property is instrumental for using Cas14 to detect single nucleotide polymorphisms (SNPs) in DNA. Using Cas14 in the DETECTR platform permitted identification of SNPs in human HERC2 gene, which is responsible for eye coloring ${ }^{44}$.

Another group took advantage of Cas12a protein to develop the one-hour lowcost multipurpose highly efficient system (HOLMES) system ${ }^{58}$. Instead of isothermal amplification used by the DETECTR method for enriching target nucleic acids, HOLMES uses PCR. Using HOLMES, the authors detected DNA viruses like Japanese encephalitis virus and Aujeszky's disease virus in 1-10 attomolaramounts ${ }^{58}$. However, the need for PCR amplification requires expensive equipment and adds an additional, time-consuming step.

The modified HOLMESv2 method uses isothermal amplification of nucleic acids by LoopMediated Isothermal Amplification (LAMP) and recognition of target double-stranded or singlestranded DNA templates by Cas12b protein followed by degradation of single-stranded DNA fluorescent probes by Cas12b collateral activity ${ }^{59}$. Peak collateral activity is reached within 10 minutes after recognition of target single-stranded DNA molecules by Cas12b, and shifts to 30 minutes when Cas12b recognizes double-stranded DNA. This property indicates the intricate differences between Cas12b and Cas12a, with the former protein being more active toward double-stranded DNA. Alicyclobacillus acidoterrestrisCas12b (AacCas12b) recognizes and cleaves target double-stranded DNA with adjacent PAM sequence 5'-TTN-3' and exhibits collateral activity ${ }^{60}$. Among target templates with different PAM regions, only double-stranded DNA with PAM 5'-TTC-3' and 5'-TAC3 'sequences after cutting activates Cas12b collateral activity and the associated degradation of fluorescent DNA probes, indicating that not all double-stranded DNA sites recognized by Cas $12 \mathrm{~b}$ can effectively turn on collateral activity of the gene-editing protein. Upon binding to single-stranded DNA, collateral activity of Cas12b was shown to be PAM-independent, always activated after interaction with an appropriate target. The lowest concentration of the target single-stranded or double-stranded DNA detected by Cas $12 \mathrm{~b}$ was around $1 \mathrm{nM}$. Combining Cas12b and LAMP technologies provides specific detection of as little as 0,01 fM DNA, which is equivalent to the sensitivity of Cas12a. HOMLESv2allows detection of trace amounts of both DNA and RNA as well, if the isolate is reverse transcribed during sample preparation. To reduce the time needed to perform RNA reverse transcription and amplification, the authors proposed using DNA polymerases with $5^{\prime} \rightarrow 3^{\prime}$ DNA polymerization and reverse transcriptase activity (e.g., DNA polymerase Bst) ${ }^{61}$.

In 2017, Feng Zhang and colleagues presented SHERLOCK, a diagnostic platform based on CRISPR-Cas type VI system ${ }^{16,62}$. SHERLOCK is based on the same principles as DETECTR, but relies on activity of Cas13 nuclease from Leptotrichia wadei. Cas13 specifically recognizes and cleaves only RNA, rather than DNA like Cas12a, thus non-specifically degrading any neighboring RNA molecules. In vitro transcription of the isolate enables detection of DNA targets. Isothermal amplification RPA can be used to enrich target molecules and increase sensitivity. The amplified RNA fragments are mixed with Cas13 protein crRNA and fluorescent RNA probes. If the target molecules are present in the sample, Cas13 recognizes them via crRNA and indiscriminately cleaves (by collateral activity) fluorescent RNA probes, disrupting the interaction between the fluorophore and the quencher. The presence and intensity of the fluorescent signal thus indicate the amount of the target in the biological sample. The authors demonstrated that SHERLOCK detects Zika virus, dengue virus, various pathogenic bacteria, and SNPs in DNA with attomolar sensitivity. All components of the SHERLOCK reaction can be lyophilized and used after long storage periods without impacting the sensitivity and specificity of the test ${ }^{16}$. SHERLOCK had a major drawback: it was qualitative, not quantitative, but a year later, the authors presented the second version of the platform, SHERLOCKv263. SHERLOCKv2 can simultaneously detect targets in 4 different fluorescent channels, because Cas13 proteins from different organisms -- LwaCas13a (L. wadei), CcaCas13b (Apnocytophaga canimorsus Cc5), LbaCas13a (L. bacterium[strain NK4A179]), and PsmCas13b (Prevotella sp. MA2016)) --destroy adjacent RNA and DNA molecules preferentially at certain dinucleotide sites (AU, UC, AC, and GA, correspondingly). Generating probes enriched in different dinucleotides and linked to different fluorophores, the authors demonstrated the ability of SHERLOCKv2 to detect up to 4 targets. 
SHERLOCKv2 also qualitatively evaluates samples with the sensitivity of 2 attomols, optimized to result in strong correlation between signal intensity and concentration of the target. The sensitivity of the signal was dramatically increased over that of SHERLOCK by adding Csm6 protein to the reaction mix. Amplification of the signal becomes possible because collateral activity of LwaCas13a and PsmCas13b destroys RNA, generating hydroxylated 5'-ends and linear homopolymers of adenine terminating with 2',3'-cyclic phosphates. The latter activates Csm6, which destroys new probe molecules and amplifies the specific signal. By testing Csm6 proteins from many different species, the authors demonstrated that Csm6 from Enterococcus italicus and Lactobacillus salivarius were most efficiently activated by 2',3'-cyclic phosphate ends. Finally, SHERLOCKv2 was engineered to produce a visual colorimetric readout on commercial lateral flow strips that do not require any special equipment. In this setting, the presence of the target is determined by visually inspecting the strips with different intensity of staining. The entire SHERLOCKv2 reaction is performed in a single step by directly applying the biological sample to the test strip without purifying and isolating nucleic acids. To conclude, SHERLOCKv2 is a highly sensitive quantitative diagnostic platform suitable for multiplex signal detection and visual/colorimetric detection on lateral flow strips ${ }^{63}$.

Myhrvold and colleagues paired the SHERLOCK protocol with a heating unextracted diagnostic samples to obliterate nuclease (HUDSON) method, aimed at detecting nucleic acids while avoiding the time-consuming step of nucleic acid isolation ${ }^{64}$. The HUDSON protocol was added to create fielddeployable diagnostics, necessary in remote areas without developed infrastructure and laboratory diagnostics. Recent outbreaks of viral infections in Africa, South America and China highlight the urgent need for developing diagnostic tests that require minimal steps, reagents, and equipment. By heating, chemically inactivating ribonucleases, and lysing viral particles, HUDSON permits direct analysis of viral pathogens in whole blood, plasma, serum, urine, or saliva samples. HUDSON can be used for virtually any CRISPR-based diagnostic platform, permitting direct analysis of viral pathogens from bodily fluids.

Table 2. Types of CRISPR-Cas-based diagnostic tools, their applications and characteristics.

\begin{tabular}{|c|c|c|c|c|c|c|c|}
\hline $\begin{array}{c}\text { Type of } \\
\text { CRISPR } \\
\text { system }\end{array}$ & Method & Protein & Target & $\begin{array}{c}\text { Amplificati } \\
\text { on }\end{array}$ & Detection & $\begin{array}{c}\text { Model } \\
\text { organism }\end{array}$ & $\begin{array}{c}\text { Reporte } \\
\text { d } \\
\text { sensitivi } \\
\text { ty }\end{array}$ \\
\hline \multirow{8}{*}{ Type II } & $\begin{array}{c}\text { Chimeric dCas9- } \\
\text { luciferase }^{51}\end{array}$ & dCas & DNA & PCR & $\begin{array}{c}\text { Luminescenc } \\
\mathrm{e}\end{array}$ & $\begin{array}{c}M . \\
\text { tuberculosis }\end{array}$ & $\begin{array}{c}\approx 3 \times 10^{-} \\
{ }^{21} \mathrm{M}\end{array}$ \\
\hline & dCas9 (FISH) ${ }^{65}$ & dCas9 & DNA & - & Fluorescence & S. aureus & $\begin{array}{c}10 \\
\text { CFU/mL }\end{array}$ \\
\hline & $\operatorname{ctPCR}^{52}$ & Cas9 & DNA & PCR & $\begin{array}{l}\text { Electrophores } \\
\text { is }\end{array}$ & HPV 16/18 & $\begin{array}{c}\approx 7 \times 10^{-} \\
{ }^{16} \mathrm{M}\end{array}$ \\
\hline & NASBACC ${ }^{15}$ & Cas9 & DNA & NASBA & Colorimetry & $\begin{array}{c}\text { Zika virus, } \\
\text { dengue virus }\end{array}$ & $\begin{array}{c}1 \times 10^{-15} \\
\mathrm{M}\end{array}$ \\
\hline & CRISPR-Chip ${ }^{53}$ & Cas9 & DNA & - & $\begin{array}{c}\text { Potentiometr } \\
\mathrm{y}\end{array}$ & SNPs & $\begin{array}{c}1.7 \times 10^{-} \\
15 \mathrm{M}\end{array}$ \\
\hline & CRISDA $^{54}$ & $\begin{array}{c}\text { Cas9 } \\
\text { nickases }\end{array}$ & DNA & SDA & Fluorescence & SNPs & $\begin{array}{l}\approx 10^{-18} \\
10^{-17} \mathrm{M}\end{array}$ \\
\hline & FLASH $^{55}$ & Cas9 & DNA & PCR & NGS & $\begin{array}{l}\text { Antimicrobi } \\
\text { al resistance } \\
\text { genes }\end{array}$ & $\begin{array}{l}\approx 10^{-18} \\
10^{-17} \mathrm{M}\end{array}$ \\
\hline & CAS-EXPAR $^{56}$ & Cas9 & $\begin{array}{l}\text { DNA, } \\
\text { RNA }\end{array}$ & EXPAR & Fluorescence & $\begin{array}{c}\text { L. } \\
\text { monocytoge } \\
\text { nis }\end{array}$ & $\begin{array}{c}0.82 \times \\
10^{-18} \mathrm{M}\end{array}$ \\
\hline \multirow{3}{*}{ Type V } & DETECTR $^{48}$ & Cas12a & DNA & RPA & Fluorescence & HPV 16/18 & $\begin{array}{l}\approx 10^{-18} \\
10^{-17} \mathrm{M}\end{array}$ \\
\hline & HOLMES $^{58}$ & Cas12a & $\begin{array}{l}\text { DNA, } \\
\text { RNA }\end{array}$ & PCR & Fluorescence & $\begin{array}{c}\text { Japanese } \\
\text { encephalitis } \\
\text { virus, } \\
\text { pseudorabie } \\
\text { s virus } \\
\end{array}$ & $\begin{array}{l}\approx 10^{-18} \\
10^{-17} \mathrm{M}\end{array}$ \\
\hline & HOLMESv2 $2^{59}$ & Cas $12 b$ & $\begin{array}{l}\text { DNA, } \\
\text { RNA }\end{array}$ & LAMP & Fluorescence & $\begin{array}{c}\text { Japanese } \\
\text { encephalitis } \\
\text { virus }\end{array}$ & $10^{-17} \mathrm{M}$ \\
\hline
\end{tabular}




\begin{tabular}{|c|c|c|c|c|c|c|c|}
\hline & E-CRISPR $^{66}$ & Cas12a & $\begin{array}{l}\text { DNA, } \\
\text { protein }\end{array}$ & - & $\begin{array}{l}\text { Electrochemi } \\
\text { cal }\end{array}$ & $\begin{array}{c}\text { DNA: } \\
\text { HPV16, } \\
\text { parvovirus } \\
\text { B19; } \\
\text { Protein: } \\
\text { TGF } \beta 1 \\
\end{array}$ & $10^{-12} \mathrm{M}$ \\
\hline Type VI & SHERLOCK $^{16,62}$ & Cas13a & $\begin{array}{l}\text { DNA, } \\
\text { RNA }\end{array}$ & RPA & Fluorescence & $\begin{array}{c}\text { Viruses, } \\
\text { bacteria, } \\
\text { SNPs }\end{array}$ & $\begin{array}{c}2 \times 10^{-18} \\
\mathrm{M}\end{array}$ \\
\hline $\begin{array}{c}\text { Type } \\
\text { V+Type } \\
\text { VI+Type } \\
\text { III }\end{array}$ & SHERLOCKv $2^{63}$ & $\begin{array}{c}\text { Cas13, } \\
\text { Cas } 12 \mathrm{a}, \\
\text { Csm6 }\end{array}$ & $\begin{array}{l}\text { DNA, } \\
\text { RNA }\end{array}$ & RPA & $\begin{array}{c}\text { Fluorescence, } \\
\text { lateral flow } \\
\text { assay }\end{array}$ & $\begin{array}{l}\text { Viruses, } \\
\text { bacteria, } \\
\text { SNPs }\end{array}$ & $\begin{array}{c}8 \times 10^{-21} \\
\mathrm{M}\end{array}$ \\
\hline
\end{tabular}

Similarly, the HUDSON-SHERLOCK pipeline can detect several targets in one clinical sample ${ }^{64}$. Using pre-defined biological samples, the authors demonstrated that the test detected Dengue virus with high sensitivity (limit of detection was 45 copies of the viral genomes in $1 \mathrm{~mL}$ of whole blood and 1 copy of the viral genome in $1 \mathrm{~mL}$ of saliva) with the reported total turnaround time of less than 1 hour ${ }^{64}$. Because viral diversity may affect sensitivity and specificity of the test, the authors then designed SHERLOCK crRNAs to discriminate between Zika virus and dengue virus. HUDSONSHERLOCK successfully differentiated 4 serotypes of dengue and Zika viruses in biological samples from different regions of the world, mostly with $100 \%$ specificity, $100 \%$ sensitivity, and $100 \%$ concordance between the samples ${ }^{64}$.

Dai and colleagues created E-CRISPR system is equipped with electrochemical signal detection module utilizing Cas12a trans-cleavage activity and modified ssDNA reporter with methylene blue electrochemical tag attached to the sensor surface ${ }^{66}$. In the presence of target sequence, Cas12a proteins cleave ssDNA-MB reporter decreasing the level of electrochemical signal being transduced. The authors achieved picomolar sensitivity for detecting HPV16 and parvovirus B-19 nucleic acids. Moreover, authors devised an aptamer-based E-CRISPR cascade for protein detection. The platform utilizes aptamers for the protein of interest and Cas12a-crRNA designed to specifically target the aptamer. E-CRISPR is applied to evaluate the remaining concentration of aptamer in the sample. The authors confirmed the efficacy of the platform by TGF- $\beta 1$ protein detection in clinical samples. Hence, E-CRISPT technology can potentially be used for wide variety of nucleic acid and proteins.

A plethora of new CRISPR-based diagnostic tools and approaches has been devised so far, but most of the early inventions relied on CRISPR-Cas9 type II systems, which themselves do not induce a strong, specific signal related to the presence of target nucleic acids in the sample. Instead, these technologies utilized pre-amplification of nucleic acids using PCR, an approach that compromises the vast perspectives of CRISPR diagnostics. More complicated methods, such as CRISDA ${ }^{54}$, work in completely isothermal conditions, but are expensive (requiring many enzymes) and time-consuming (total turnaround time for CRISDA is over 3-4 hours). Although several methods, such as FLASH ${ }^{55}$, can be applied in certain specific areas of molecular diagnostics, the majority of technologies listed in Table 2 do not demonstrate any obvious advantages over PCR. 


\section{DETECTR}

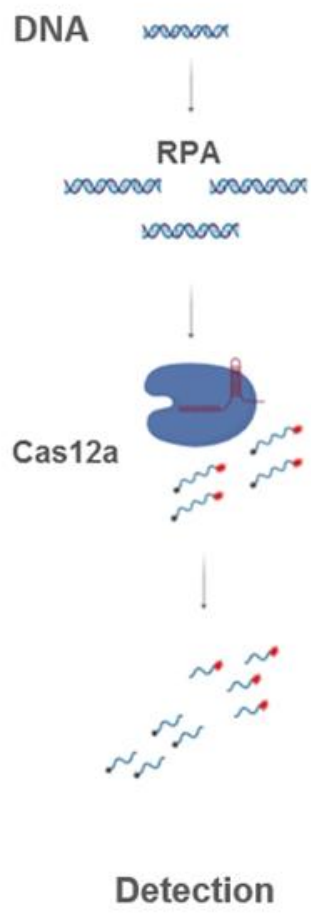

\section{SHERLOCK / SHERLOCKv2}

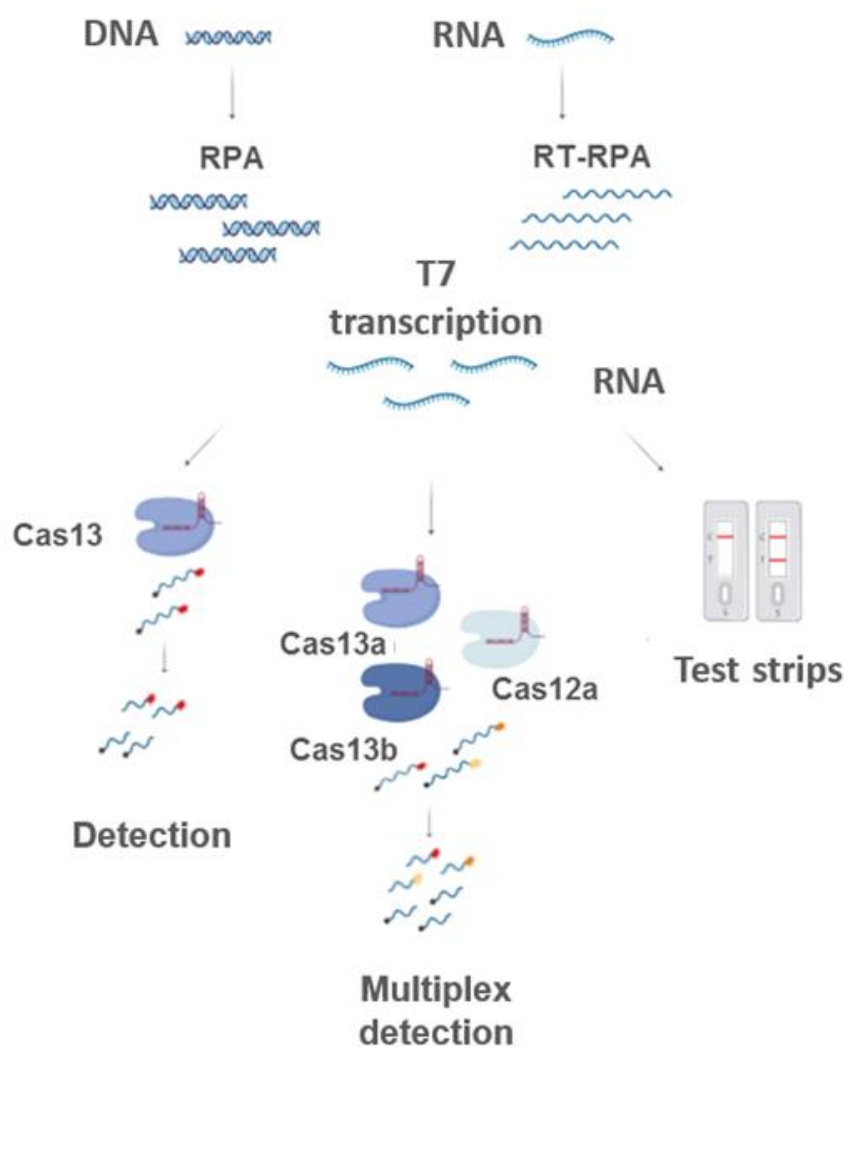

Figure 1. Schematics of CRISPR-Cas diagnostic platforms DETECTR and SHERLOCK/v2.

In contrast, DETECTR, SHERLOCK/SHERLOCKv2, and CRISPR-Chip can be considered breakthroughs in molecular diagnostics, representing almost ideal diagnostic tests that can be used in completely isothermal conditions, with minimal equipment and hands-on training of the personnel. Workflow of DETECTR and SHERLOCK/v2 is described in Fig. 1. Especially important is that SHERLOCKv2 is already adopted for colorimetric and visual detection of pathogens on lateral flow strips, so that these tests can be utilized for mass screening and rapid diagnostics in virtually any geographic region ${ }^{63}$. High sensitivity and specificity of CRISPR-Cas systems extend their potential application beyond qualitative and quantitative detection of different pathogens to genotyping and detecting single SNPs in human genomes.

Although the described works provide exciting perspectives that may grow into a huge area of research and molecular diagnostics, many challenges stand in the way. Very encouraging results demonstrating specificity and sensitivity of assays similar to PCR should be tested in real-world situations. Many modifications to existing assays may be introduced to increase sensitivity of the tests and time required to perform diagnostic tests. Moreover, specificity of CRISPR diagnostics may also be a concern, as Cas proteins can tolerate nucleotide mismatches and recognize non-specific templates, compromising assay validity. It is also not quite clear how discrepant the results of such tests could be when handled by different personnel or with varying qualities of biological samples.

\section{PCR v. CRISPR-Cas}


Understanding the pros and cons of using CRISPR-Cas and PCR is important for defining the scope, advantages, and downsides of each method. Large-scale testing and comparative analysis of different PCR-based and CRISPR diagnostic systems in the real world is mandatory to move new methods into laboratory and field diagnostics. Introducing CRISPR diagnostic tools into practice may improve control over infectious diseases, prevent their rapid spread, introduce diagnostic testing to finally enter the era of precise and personalized medicine ${ }^{67}$, expand the use of massive population screening, and better control outbreaks of infectious diseases, including outbreaks in remote geographic areas. Timely, inexpensive, high-quality diagnosis of infectious and non-infectious diseases by rapid and readily available tests will certainly have a pronounced positive impact on human health on the global level, reducing morbidity and mortality.

Amplification of nucleic acids by PCR, the central dogma of molecular diagnostics, has long been the only practical way to detect trace amounts of infectious pathogens in biological samples. PCR analysis requires expensive equipment and qualified personnel to run the tests and interpret the results. Many attempts have been undertaken in the past years to avoid PCR cycling and adopt amplification to isothermal conditions. However, all other methods (NASBA, LAMP, RPA, HAD) ${ }^{68}$ suffered from serious flaws compromising their utility in molecular diagnostics; these flaws included low sensitivity, low specificity, complicated reactions, and high price. Embedding methods of isothermal amplification into CRISPR diagnostic platforms eliminates the shortcomings of both technologies, achieving rapid and highly specific results. Compared to PCR, SHERLOCK/SHERLOCKv2 and DETECTR add another level of specificity because these methods not only use specific primers during isothermal amplification, but also specifically recognize target templates via Cas-sg/crRNA complex.

Another important property to consider is the ability of CRISPR proteins to tolerate nucleotide mismatches between sg/crRNA and the target template ${ }^{13}$. PCR is very sensitive to mismatched nucleotides at the 3 '-end of primers, which dramatically reduces non-specific primer annealing and ensures accurate target amplification ${ }^{69}$. CRISPR proteins recognize and bind degenerate sequences with multiple mismatches, depending on the nature of the CRISPR protein. On the one hand, this property may compromise the validity of diagnostic tests, leading to false positive results. On the other, ignoring nucleotide mismatches allows CRISPR proteins to recognize naturally variable genetic sequences. For instance, this property may facilitate effective detection of genetically distinct mutant HIV genomes, hepatitis $\mathrm{C}$ genomes, etc. However, this property may be of practical use only if the CRISPR diagnostic platform is not coupled with an isothermal amplification step. Isothermal amplification with specific primers nullifies this property of CRISPR proteins by specifically amplifying totally matched genetic templates. Notably, SHERLOCKv2 can avoid isothermal amplification ${ }^{63}$. The specific signal is amplified by a combination of Cas13a proteins and co-activating Csm6 proteins, which react to cyclic oligonucleotides generated after specific cleavage of the target.

Important properties of CRISPR diagnostics are simplicity, stability of components, no requirement for isolation of nucleic acids from biological samples (in the most advanced technologies), and isothermal conditions of each step, requiring minimal equipment.

Overall, CRISPR diagnostics will not completely replace PCR in daily laboratory diagnostics in the near future, but may fill gaps in the global health care system, providing an opportunity for mass population screening, better control of infectious outbreaks, wider distribution of diagnostic techniques, and field-deployable diagnostics. Still, rapid development of the CRISPR field and identification of novel CRISPR systems with new, exciting properties will dictate the development of CRISPR diagnostics -- and laboratory diagnostics in general -- in the coming years.

\section{Potential applications of CRISPR diagnostics}

As was briefly mentioned, the ideal diagnostic assay should provide accurate and sensitive identification of the pathogen while being affordable, portable, and able to distinguish different variants of the pathogen. Currently, no such test exists. Developing new tools and methods which meet (or attempt to meet) the requirements of the WHO ideal diagnostic test can completely reshape 
epidemiological surveillance and medical health care system for the majority of infectious and noninfectious diseases in the world ${ }^{70}$.

Highly specific DNA-binding nucleases provide the promising platform to create new diagnostic tests for rapid and inexpensive detection of infectious agents or genetic mutations. A combination of CRISPR proteins and isothermal methods of amplification, such as NASBA or RPA, may enter laboratory and field diagnostics in the very near future. Novel CRISPR-diagnostic tools, their targets and applications to detect viral pathogens are summarized in Table 3.

\subsection{CRISPR-tools for socially significant infectious diseases}

Below we review some of the most socially significant infectious diseases the treatment of which would significantly improve if portable and affordable CRISPR-diagnostic platforms enter the practice. In the world, morbidity from such infectious diseases as tuberculosis, HIV, and viral hepatitis, and the epidemiological situation associated with the burden of these diseases, can be greatly reduced by currently available therapeutics if CRISPR diagnostics finally come to the stage.

Tuberculosis is an airborne infectious disease caused by M. tuberculosis. According to the latest data, more than one quarter of the world's population is infected. Causing over 1.3 million deaths per year, tuberculosis remains one of the leading infectious causes of death worldwide ${ }^{71}$. Real-time PCR analysis is a rapid and accurate method for diagnosing $M$. tuberculosis infection ${ }^{72}$. Moreover, PCR is widely used to detect drug resistance ${ }^{73}$. Zhang et al. created a new CRISPR-based approach for detecting $M$. tuberculosis DNA in clinical sample ${ }^{51}$.

HIV infection remains to be a major public health issue. More than 37,9 million people worldwide are infected ${ }^{8}$, many in developing countries with limited access to highly active antiretroviral therapy (HAART) regimens ${ }^{74}$. Early diagnosis can prevent further spread of the infection, so a new, rapid assay detecting HIV RNA in patients' blood samples is urgently needed.

Approximately 2.4 of 100,000 women die annually due to cervical cancer caused by HPV 75 . The gold standard for HPV diagnosis is viral DNA detection ${ }^{76}$ and PAP testing ${ }^{77}$. There are many types of the virus, some of them characterized by their association with cervical cancer as highly oncogenic; HPV16 and HPV18 are responsible for most HPV-related cancers. Two CRISPR systems -- CARP78and DETECTR ${ }^{48-}$ - were created for HPV diagnostics. DETECTR enables sensitive, specific, and robust detection of the virus at attomolar concentrations and allows differentiation of viral subtypes.

Viral hepatitis B, D and C affect millions of people around the world ${ }^{7,79}$. Hepatitis B virus (HBV), $\mathrm{HBV}+$ hepatitis D virus (HDV) co-infection and hepatitis $\mathrm{C}$ virus $(\mathrm{HCV})$ infection is one of the principal causes of death among infectious viral diseases ${ }^{80}$; over 1.3 million people die annually due to consequences of HBV, HBV-HDV co-infection and HCV infection. Chronic viral hepatitis is generally asymptomatic until advanced liver injury develops, and more than $80 \%$ of patients are unaware that they are infected. In low- and middle-income regions, most infected people are still not diagnosed, and many HBV, HBV-HDV co-infection and HCV carriers do not receive antiviral therapy ${ }^{81}$. No CRISPR-based diagnostic tests to detect HBV, HDV and HCV are yet available. Rapid, sensitive, and specific point-of-care assays are urgently needed to detect viral DNA or RNA in blood samples, genotype the virus, and reveal drug resistance.

\subsection{CRISPR-tools for emerging and re-emerging infectious diseases}

Three million people are estimated to be at risk of dengue fever ${ }^{82}$, caused by Dengue virus that is transmitted by mosquitos and widespread in Latin America and Asia ${ }^{83}$. Dengue fever symptoms resemble those of hemorrhagic fevers caused by Ebola virus and other viruses ${ }^{84}$. Thus, laboratory diagnosis of dengue fever is crucial for rapid management of patients with severe infection ${ }^{85}$. Dengue infection can be confirmed by isolating the virus and immunologically detecting viral protein Ns $1{ }^{86}$. Real-time PCR analysis is widely used for Ebola virus diagnostics, but expensive equipment and well-trained personnel are required to obtain accurate results ${ }^{87}$. Most importantly, the situation in the areas of outbreaks requires urgent action to identify infected persons and curb disease spread. In this respect, PCR technologies are inferior to CRISPR-tools in terms of time required to get the results and that they cannot be readily deployable for field diagnostics, especially in remote areas. Developing 
new diagnostic tools will help to prevent further spread of infection and better inform patient treatment. The CRISPR based detection system NASBACC ${ }^{15}$ can detect dengue virus, but SHERLOCKv $2^{63}$ is the most sensitive system that differentiates the four viral serotypes with high efficacy.

Zika virus is transmitted by mosquitos and has been reported to lead to neurological defects in newborns whose mothers were infected during pregnancy. Accurate and rapid diagnosis is critical for timely treatment ${ }^{88}$. Two approaches were utilized for detecting the disease: NASBACC ${ }^{15}$ and SHERLOCKv2 ${ }^{63}$. SHERLOCKv2 is more sensitive and robust, enabling femtomolar detection of the virus.

Coronaviral infections represent adaunting threat to the global health, appearing every 10-20 years with new outbreaks, diseasing thousands of people with relatively high death toll. The first case of severe acute respiratory syndrome (SARS) has been documented in November 2002 in Foshan, China ${ }^{89}$. During four months the disease spread into 27 countries, and a total of 8,096 cases were identified ${ }^{90}$.Since the outbreak, only the sporadic cases were reported. Bats are known to be a natural reservoir for a wide range of coronaviruses, but human-bat interactions are relatively rare, so an intermediate host is thought to be required to infect humans. However, it has been recently demonstrated that coronaviruses directly isolated from bats are able to infect human cells without prior adaptation ${ }^{91,92}$. These data indicate that novel infections can emerge potentially leading to widespread outbreaks ${ }^{5}$. Middle East respiratory syndrome virus (MERS-CoV) was isolated from sputum of a man who died of acute pneumonia and renal failure in $2012{ }^{93}$. By April 2016, MERS has been detected in 27 countries, and 1,728 infection cases were confirmed ${ }^{94}$. MERS-CoV-like viruses are shown to inhabit dromedary camels ${ }^{5,95}$. Thus, MERS-CoV zoonotic transmission is not rare, and MERS-CoV continues to infect humans due to the fact that dromedary camel are farmed and are in close proximity to humans ${ }^{5}$.Both SARS and MERS viruses have high risk of nosocomial transmission 96,97 and were spread from the initial outbreak location by infected individuals ${ }^{98-101}$. PCR assays have been developed for SARS and MERS CoVs detection 102,103. New human Coronavirus infection emerged in December 2019 in Wuhan, China, and currently it is an ongoing outbreak that spread through the mainland of China and neighboring countries ${ }^{6}$. The first cases of novel coronavirus infection were reported in a fresh seafood market which distributed various mammals and exotic wildlife animals, including bats, snakes, rats, beavers etc. Such areas of crowded people, exotic wildlife and low-quality sanitary conditions represent high-risk places for emerging of novel zoonotic infections. The symptoms of the novel viral infection were identified as fever, dry cough, headache and pneumonia ${ }^{104}$. The infectious agent was identified as Coronavirus, designated as 2019$\mathrm{nCoV}$. At the current time over 2,000 confirmed cases have been reported and over 40 people died due to infection ${ }^{105}$.

Table 3. Infectious agents as diagnostic targets for CRISPR-Cas-diagnostics.

\begin{tabular}{ccc}
\hline Pathogen & Target & CRISPR-diagnostic \\
\hline Tuberculosis & Socially significant infectious diseases & \\
\hline HIV & DNA, antibiotic resistance testing & Chimeric dCas9 luciferase \\
\hline HPV & RNA & - \\
\hline HBV & DNA, virus typing & ctPCR, DETECTR \\
\hline \multirow{2}{*}{ HBV-HDV co-infection } & DNA, genotyping & - \\
& RNA, genotyping & - \\
\hline \multirow{2}{*}{ HCV } & RNA, genotyping, drug resistance testing & - \\
\hline \multicolumn{4}{c}{ Emerging and re-emerging infectious diseases } \\
\hline SARS-CoV & RNA & - \\
\hline MERS-CoV & RNA & - \\
\hline nCov-2019 & RNA & - \\
\hline Dengue virus & RNA & SHERLOCK, SHERLOCKv2 \\
\hline Ebola virus & RNA & SHERLOCK, SHERLOCKv2 \\
\hline Zika virus & RNA &
\end{tabular}


Clinical features of pneumonia caused by 2019-nCov were described shortly after the outbreak. Full-genome sequencing of 2019-nCov revealed that it belongs to group 2B of the Betacoronaviridae family, possessing $70 \%$ similarity to SARS-CoV. The source of the virus still remains unknown, although an initial report suggested snakes as possible intermediate hosts ${ }^{106}$. Nevertheless, another scientific group reported that virus potentially has a bat origin ${ }^{107}$. Due to the rapid spread of the virus, express diagnostics is essential for reducing the risk of further transmission. CRISPR based diagnostic tests would be helpful for effective identification, diagnosis and management of the infection. Shortly after the outbreak and sequencing of the virus, PCR-system for detecting the pathogen was developed. However, PCR does not fit the relevant needs to control and curb the emerging outbreaks, as is noted throughout the review. The ideal tool would have been an express-method to unequivocally detect the pathogen and identified infected persons. Although the field of CRISPRdiagnostics is at the early stage of development, further advancement of such tests in the expressformat holds a lot of promise both in the daily practice (point-of-care tests) and in epidemiological surveillance programs to prevent disease spread. Notably, CRISPR-systems have not yet been utilized to detect coronaviruses.

Field-deployable diagnostic tests that have low turnaround time and could be rapidly distributed for timely detection of viral pathogens can prevent global and regional spread given extremely rapid transportation systems and high contagiosity of emerging viral pathogens. Frequent (every 20-30 years, as reported by far) emergence of novel, highly contagious viral infections, proves the urgent need for developing field-deployable diagnostics which could have a profound effect on identification of new infectious cases and prevention of disease spread.

Rapid pathogen detection is a crucial part of molecular diagnostic. Recently described CRISPRbased methods for HPV, Zika virus, and dengue virus detection demonstrated high potency for using in diagnostic field. Although standard methods of nucleic acid amplification are highly effective, they require expensive instrumentation and well-trained lab personnel. Point-of-care assays based on CRISPR are a versatile detection platform for clinical diagnosis of infectious diseases, enabling rapid and robust detection of pathogens in limited-resource areas.

\section{Conclusion}

Although many milestones have been surpassed within several years, CRISPR diagnostics is still at the dawn of its development. Numerous obstacles may still hamper rapid translation of laboratory results into practice, including off-target recognition and potential false-positive results associated with the intricate property of CRISPR proteins to tolerate mismatched nucleotides. Designing software-backed optimal sgRNAs with minimal potential off-target effects (e.g., CHOPCHOP${ }^{108}$ ), and identifying or inventing novel CRISPR proteins with more practical properties or limited mismatch tolerance may solve this potential problem. Cas proteins can currently be adjusted by, for instance, direct evolution to change PAM sequence requirements (shorter/longer PAMs or PAMs with certain nucleotide preference ${ }^{36,39}$ ) or higher specificity (e.g., enSpCas $9^{30}$ ).Sg/crRNAs have also undergone many modifications, starting from shortened sg/crRNAs ${ }^{109}$ to $\mathrm{sg} / \mathrm{crRNAs}$ with engineered RNA hairpins ${ }^{110}$. Predicting and identifying novel CRISPR systems is currently the hottest topic in biology. One of the many examples of successful identification of CRISPR proteins with previously unknown properties is Cas13a (previously referred to as C2c2), which has transformed into a powerful molecular tool ${ }^{16}$. Application of CRISPR may go far beyond detection of nucleic acids, as several CRISPR technologies have been devised to identify proteins ${ }^{66}$ and could be utilized for detecting small molecules ${ }^{111}$ (e.g. toxic agents, opioids, drugs etc.). Practically, they may transform into new, more efficient and cheap approaches that could replace many conventional techniques, such as immunosorbent assays, chromatography etc.

Potential challenges mostly include the low quality of clinical sample, when specificity or sensitivity of the test could be compromised by the abundance of reaction inhibitors and interfering compounds. It is also unclear what types of the samples could be used for CRISPR-diagnostics, such as dry blood spot cards etc. Automation and high-throughput use of CRISPR-screening has not yet been addressed. Most importantly, CRISPR-diagnostics may provide the means for rapid, express- 
diagnostics, but the time currently required for performing the CRISPR-mediated identification of target nucleic acids is still far from being considered an «express method».

To conclude, using CRISPR-based methods to detect and qualitatively analyze infectious pathogens and more is a new reality in the field of molecular diagnostics. Developing new CRISPR tools and platforms for molecular diagnosis promises to reshape health care and improve epidemiological management on a global level.

Funding: This work was performed within RFBR grant no. 20-015-00442.

Conflicts of Interest: The authors declare no conflict of interests.

\section{References}

1. Hwang, H., Hwang, B.-Y. \& Bueno, J. Biomarkers in Infectious Diseases. Disease markers 2018, 8509127 (2018).

2. Kilbourne, E. D. Influenza pandemics of the 20th century. Emerg. Infect. Dis. 12, 9 (2006).

3. Moon, S. et al. Will Ebola change the game? Ten essential reforms before the next pandemic. The report of the Harvard-LSHTM Independent Panel on the Global Response to Ebola. Lancet 386, 2204-2221 (2015).

4. Gatherer, D. \& Kohl, A. Zika virus: a previously slow pandemic spreads rapidly through the Americas. J. Gen. Virol. 97, 269-273 (2016).

5. De Wit, E., Van Doremalen, N., Falzarano, D. \& Munster, V. J. SARS and MERS: Recent insights into emerging coronaviruses. Nat. Rev. Microbiol. 14, 523-534 (2016).

6. Cohen, J. \& Normile, D. New SARS-like virus in China triggers alarm. (2020).

7. World Health Organization. Global hepatitis report, 2017. Who (2017). doi:ISBN 978-92-4-156545-5

8. UNAIDS. AIDS statistics-2018 fact sheet. UNAIDS website. unaids. org/en/resources/fact-sheet. Accessed May 31, (2019).

9. Watzinger, F., Ebner, K. \& Lion, T. Detection and monitoring of virus infections by real-time PCR. Mol. Aspects Med. 27, 254-298 (2006).

10. Shalhoub, S. et al. IFN- $\alpha 2$ a or IFN- $\beta 1$ a in combination with ribavirin to treat Middle East respiratory syndrome coronavirus pneumonia: a retrospective study. J. Antimicrob. Chemother. 70, 2129-2132 (2015).

11. Peeling, R. W., Holmes, K. K., Mabey, D. \& Ronald, A. Rapid tests for sexually transmitted infections (STIs): the way forward. Sex. Transm. Infect. 82, v1-v6 (2006).

12. Kettler, H., White, K. \& Hawkes, S. J. Mapping the landscape of diagnostics for sexually transmitted infections: key findings and recommendations. (Geneva: World Health Organization, 2004).

13. Jiang, W., Bikard, D., Cox, D., Zhang, F. \& Marraffini, L. A. RNA-guided editing of bacterial genomes using CRISPR-Cas systems. Nat. Biotechnol. 31, 233-239 (2013).

14. Riordan, S. M., Heruth, D. P., Zhang, L. Q. \& Ye, S. Q. Application of CRISPR/Cas9 for biomedical discoveries. Cell Biosci. 5, 33 (2015).

15. Pardee, K. et al. Rapid, Low-Cost Detection of Zika Virus Using Programmable Biomolecular Components. Cell 165, 1255-1266 (2016).

16. Gootenberg, J. S. et al. Nucleic acid detection with CRISPR-Cas13a/C2c2. Science 356, 438-442 (2017).

17. Ishino, Y., Shinagawa, H., Makino, K., Amemura, M. \& Nakata, A. Nucleotide sequence of the iap gene, responsible for alkaline phosphatase isozyme conversion in Escherichia coli, and identification of the gene product. J. Bacteriol. 169, 5429-5433 (1987).

18. Haft, D. H., Selengut, J., Mongodin, E. F. \& Nelson, K. E. A guild of 45 CRISPR-associated (Cas) protein families and multiple CRISPR/Cas subtypes exist in prokaryotic genomes. PLoS Comput. Biol. 1, e60 (2005).

19. Makarova, K. S. \& Koonin, E. V. Annotation and Classification of CRISPR-Cas Systems. Methods Mol. Biol. 1311, 47-75 (2015).

20. Brouns, S. J. J. et al. Small CRISPR RNAs guide antiviral defense in prokaryotes. Science 321, 960-964 (2008).

21. Hayes, R. P. et al. Structural basis for promiscuous PAM recognition in type I-E Cascade from E. coli. Nature 530, 499-503 (2016).

22. Kazlauskiene, M., Kostiuk, G., Venclovas, C., Tamulaitis, G. \& Siksnys, V. A cyclic oligonucleotide signaling pathway in type III CRISPR-Cas systems. Science 357, 605-609 (2017). 
23. Niewoehner, O. et al. Type III CRISPR-Cas systems produce cyclic oligoadenylate second messengers. Nature 548, 543-548 (2017).

24. Koonin, E. V \& Makarova, K. S. Origins and evolution of CRISPR-Cas systems. Philos. Trans. R. Soc. Lond. B. Biol. Sci. 374, 20180087 (2019).

25. Makarova, K. S. et al. An updated evolutionary classification of CRISPR-Cas systems. Nat. Rev. Microbiol. 13, 722-736 (2015).

26. Shmakov, S. et al. Diversity and evolution of class 2 CRISPR-Cas systems. Nat. Rev. Microbiol. 15, 169182 (2017).

27. Mohanraju, P. et al. Diverse evolutionary roots and mechanistic variations of the CRISPR-Cas systems. Science (80-. ). 353, aad5147 (2016).

28. Cong, L. et al. Multiplex Genome Engineering Using CRISPR/VCas Systems. Science (80-. ). 339, 819-823 (2013).

29. Jinek, M. et al. A programmable dual-RNA-guided DNA endonuclease in adaptive bacterial immunity. Science 337, 816-821 (2012).

30. Slaymaker, I. M. et al. Rationally engineered Cas9 nucleases with improved specificity. Science (80-. ). 351, 84-88 (2016).

31. Kleinstiver, B. P. et al. High-fidelity CRISPR-Cas9 nucleases with no detectable genome-wide off-target effects. Nature 529, 490-495 (2016).

32. Casini, A. et al. A highly specific SpCas9 variant is identified by in vivo screening in yeast. Nat. Biotechnol. 36, 265 (2018).

33. Ikeda, A., Fujii, W., Sugiura, K. \& Naito, K. High-fidelity endonuclease variant HypaCas9 facilitates accurate allele-specific gene modification in mouse zygotes. Commun. Biol. 2, 1-7 (2019).

34. Lee, J. K. et al. Directed evolution of CRISPR-Cas9 to increase its specificity. Nat. Commun. 9, 1-10 (2018).

35. Esvelt, K. M. et al. Orthogonal Cas9 proteins for RNA-guided gene regulation and editing. Nat. Methods 10, 1116-1123 (2013).

36. Lee, C. M., Cradick, T. J. \& Bao, G. The Neisseria meningitidis CRISPR-Cas9 System Enables Specific Genome Editing in Mammalian Cells. Mol. Ther. 24, 645-654 (2016).

37. Kostyushev, D. et al. Orthologous CRISPR/Cas9 systems for specific and efficient degradation of covalently closed circular DNA of hepatitis B virus. Cell. Mol. LIFE Sci. 76, 1779-1794 (2019).

38. Ran, F. A. et al. In vivo genome editing using Staphylococcus aureus Cas9. Nature 520, 186-191 (2015).

39. Müller, M. et al. Streptococcus thermophilus CRISPR-Cas9 Systems Enable Specific Editing of the Human Genome. Mol. Ther. 24, 636-644 (2016).

40. Amrani, N. et al. NmeCas9 is an intrinsically high-fidelity genome-editing platform. Genome Biol. 19, 214 (2018).

41. Makarova, K. S. et al. Evolutionary classification of CRISPR-Cas systems: a burst of class 2 and derived variants. Nat. Rev. Microbiol. 1-17 (2019).

42. Kim, D. et al. Genome-wide analysis reveals specificities of Cpf1 endonucleases in human cells. Nat. Biotechnol. 34, 863-868 (2016).

43. Bothmer, A. et al. Characterization of the interplay between DNA repair and CRISPR/Cas9-induced DNA lesions at an endogenous locus. Nat. Commun. 8, 13905 (2017).

44. Harrington, L. B. et al. Programmed DNA destruction by miniature CRISPR-Cas14 enzymes. Science 362, 839-842 (2018).

45. Abudayyeh, O. O. et al. RNA targeting with CRISPR-Cas13. Nature 550, 280-284 (2017).

46. Cox, D. B. T. et al. RNA editing with CRISPR-Cas13. Science 358, 1019-1027 (2017).

47. Makarova, K. S., Wolf, Y. I. \& Koonin, E. V. Classification and Nomenclature of CRISPR-Cas Systems: Where from Here? Cris. J. 1, 325-336 (2018).

48. Chen, J. S. et al. CRISPR-Cas12a target binding unleashes indiscriminate single-stranded DNase activity. Science 360, 436-439 (2018).

49. Wang, H., La Russa, M. \& Qi, L. S. CRISPR/Cas9 in Genome Editing and Beyond. Annu. Rev. Biochem. 85, 227-264 (2016).

50. Brezgin, S., Kostyusheva, A., Kostyushev, D. \& Chulanov, V. Dead Cas Systems: Types, Principles, and Applications. Int. J. Mol. Sci. 20, (2019).

51. Zhang, Y. et al. Paired Design of dCas9 as a Systematic Platform for the Detection of Featured Nucleic Acid Sequences in Pathogenic Strains. ACS Synth. Biol. 6, 211-216 (2017). 
52. Wang, Q., Zhang, B., Xu, X., Long, F. \& Wang, J. CRISPR-typing PCR (ctPCR), a new Cas9-based DNA detection method. Sci. Rep. 8, 14126 (2018).

53. Hajian, R. et al. Detection of unamplified target genes via CRISPR-Cas9 immobilized on a graphene field-effect transistor. Nat. Biomed. Eng. 3, 427-437 (2019).

54. Zhou, W. et al. A CRISPR-Cas9-triggered strand displacement amplification method for ultrasensitive DNA detection. Nat. Commun. 9, 5012 (2018).

55. Quan, J. et al. FLASH: a next-generation CRISPR diagnostic for multiplexed detection of antimicrobial resistance sequences. Nucleic Acids Res. (2019). doi:10.1093/nar/gkz418

56. Huang, M., Zhou, X., Wang, H. \& Xing, D. Clustered Regularly Interspaced Short Palindromic Repeats/Cas9 Triggered Isothermal Amplification for Site-Specific Nucleic Acid Detection. Anal. Chem. 90, 2193-2200 (2018).

57. Zhang, Y. \& Zhang, C. Sensitive detection of microRNA with isothermal amplification and a singlequantum-dot-based nanosensor. Anal. Chem. 84, 224-231 (2012).

58. Li, S.-Y. et al. CRISPR-Cas12a-assisted nucleic acid detection. Cell Discov. 4, 20 (2018).

59. Li, L. et al. HOLMESv2: A CRISPR-Cas12b-Assisted Platform for Nucleic Acid Detection and DNA Methylation Quantitation. ACS Synth. Biol. 8, 2228-2237 (2019).

60. Yan, W. X. et al. Functionally diverse type V CRISPR-Cas systems. Science (80-. ). 363, 88-91 (2019).

61. Li, L., Li, S. \& Wang, J. CRISPR-Cas12b-assisted nucleic acid detection platform. bioRxiv 362889 (2018). doi:10.1101/362889

62. Kellner, M. J., Koob, J. G., Gootenberg, J. S., Abudayyeh, O. O. \& Zhang, F. SHERLOCK: nucleic acid detection with CRISPR nucleases. Nat. Protoc. 14, 2986-3012 (2019).

63. Gootenberg, J. S. et al. Multiplexed and portable nucleic acid detection platform with Cas13, Cas12a, and Csm6. Science 360, 439-444 (2018).

64. Myhrvold, C. et al. Field-deployable viral diagnostics using CRISPR-Cas13. Science 360, 444-448 (2018).

65. Guk, K. et al. A facile, rapid and sensitive detection of MRSA using a CRISPR-mediated DNA FISH method, antibody-like dCas9/sgRNA complex. Biosens. Bioelectron. 95, 67-71 (2017).

66. Dai, Y. et al. Exploring the Trans-Cleavage Activity of CRISPR-Cas12a (cpf1) for the Development of a Universal Electrochemical Biosensor. Angew. Chemie Int. Ed. 58, 17399-17405 (2019).

67. Bodrova, T. A. et al. Introduction into PPPM as a new paradigm of public health service: an integrative view. EPMA J. 3, 16 (2012).

68. Zanoli, L. M. \& Spoto, G. Isothermal amplification methods for the detection of nucleic acids in microfluidic devices. Biosensors 3, 18-43 (2012).

69. Boyle, B., Dallaire, N. \& MacKay, J. Evaluation of the impact of single nucleotide polymorphisms and primer mismatches on quantitative PCR. BMC Biotechnol. 9, 75 (2009).

70. Peeling, R. W. Testing for sexually transmitted infections: a brave new world? Sexually transmitted infections 82, 425-430 (2006).

71. Sulis, G., Roggi, A., Matteelli, A. \& Raviglione, M. C. Tuberculosis: epidemiology and control. Mediterr. J. Hematol. Infect. Dis. 6, e2014070-e2014070 (2014).

72. Salian, N. V, Rish, J. A., Eisenach, K. D., DONALD CAVE, M. \& Bates, J. H. Polymerase chain reaction to detect Mycobacterium tuberculosis in histologic specimens. Am. J. Respir. Crit. Care Med. 158, 11501155 (1998).

73. Hristea, A. et al. Detection of Mycobacterium tuberculosis resistance mutations to rifampin and isoniazid by real-time PCR. Indian J. Med. Microbiol. 28, 211 (2010).

74. Tay, A., Pavesi, A., Yazdi, S. R., Lim, C. T. \& Warkiani, M. E. Advances in microfluidics in combating infectious diseases. Biotechnol. Adv. 34, 404-421 (2016).

75. Franco, E. L., Duarte-Franco, E. \& Ferenczy, A. Cervical cancer: epidemiology, prevention and the role of human papillomavirus infection. Cmaj 164, 1017-1025 (2001).

76. Rozendaal, L. et al. PCR-based high-risk HPV test in cervical cancer screening gives objective risk assessment of women with cytomorphologically normal cervical smears. Int. J. Cancer 68, 766-769 (1996).

77. Naucler, P. et al. Human papillomavirus and Papanicolaou tests to screen for cervical cancer. N. Engl. J. Med. 357, 1589-1597 (2007).

78. Zhang, B. et al. Detection of target DNA with a novel Cas9/sgRNAs-associated reverse PCR (CARP) technique. Anal. Bioanal. Chem. 410, 2889-2900 (2018).

79. Чуланов, В. П. et al. Гепатит С излечим: гепатит В - следующий? Ter. Arkh. 89, 4-13 (2017). 
80. Papatheodoridis, G. et al. Addressing barriers to the prevention, diagnosis and treatment of hepatitis B and $\mathrm{C}$ in the face of persisting fiscal constraints in Europe: report from a high level conference. J. Viral Hepat. 23 Suppl 1, 1-12 (2016).

81. Denniston, M. M., Klevens, R. M., McQuillan, G. M. \& Jiles, R. B. Awareness of infection, knowledge of hepatitis $C$, and medical follow-up among individuals testing positive for hepatitis C: National Health and Nutrition Examination Survey 2001-2008. Hepatology 55, 1652-1661 (2012).

82. Stanaway, J. D. et al. The global burden of dengue: an analysis from the Global Burden of Disease Study 2013. Lancet Infect. Dis. 16, 712-723 (2016).

83. Cafferata, M. L. et al. Dengue epidemiology and burden of disease in Latin America and the Caribbean: a systematic review of the literature and meta-analysis. Value Heal. Reg. issues 2, 347-356 (2013).

84. Benzine, J. W. et al. Molecular Diagnostic Field Test for Point-of-Care Detection of Ebola Virus Directly From Blood. J. Infect. Dis. 214, S234-S242 (2016).

85. Bhatt, S. et al. The global distribution and burden of dengue. Nature 496, 504-507 (2013).

86. Wang, S. M. \& Sekaran, S. D. Early diagnosis of Dengue infection using a commercial Dengue Duo rapid test kit for the detection of NS1, IGM, and IGG. Am. J. Trop. Med. Hyg. 83, 690-695 (2010).

87. Pang, J., Chia, P. Y., Lye, D. C. \& Leo, Y. S. Progress and Challenges towards Point-of-Care Diagnostic Development for Dengue. J. Clin. Microbiol. 55, 3339-3349 (2017).

88. Waggoner, J. J. \& Pinsky, B. A. Zika Virus: Diagnostics for an Emerging Pandemic Threat. J. Clin. Microbiol. 54, 860-867 (2016).

89. Zhong, N. S. et al. Epidemiology and cause of severe acute respiratory syndrome (SARS) in Guangdong, People's Republic of China, in February, 2003. Lancet 362, 1353-1358 (2003).

90. World Health Organization. Epidemic and Pandemic Alert Response: Summary of probably SARS cases with onset of illness from 1 November 2002 to 31 July 2003. Based data as 31, (2003).

91. Ge, X. Y. et al. Isolation and characterization of a bat SARS-like coronavirus that uses the ACE2 receptor. Nature 503, 535-538 (2013).

92. Menachery, V. D. et al. SARS-like cluster of circulating bat coronavirus pose threat for human emergence HHS Public Access. Nat Med 21, 1508-1513 (2015).

93. Hijawi, B. et al. Novel coronavirus infections in Jordan, April 2012: Epidemiological findings from a retrospective investigation. East. Mediterr. Heal. J. 19, 12-18 (2013).

94. Almaghrabi, R. S. \& Omrani, A. S. Middle East respiratory syndrome coronavirus (MERS-CoV) infection. Br. J. Hosp. Med. 78, 23-26 (2017).

95. Sabir, J. S. M. et al. Co-circulation of three camel coronavirus We conducted surveillance for CoVs in dromedary camels in species and recombination of MERS-CoVs Saudi Arabia, the country most affected by MERS, from May 2014 to April 2015. Initially, in Saudi Arabia. Sci. Reports 1-6 (2015).

96. Chowell, G. et al. Transmission characteristics of MERS and SARS in the healthcare setting: A comparative study. BMC Med. 13, 1-12 (2015).

97. Hunter, J. C. et al. Transmission of middle east respiratory syndrome coronavirus infections in healthcare settings, abu dhabi. Emerg. Infect. Dis. 22, 647-656 (2016).

98. Lee, N., Hul, D., Wu, A., Chan, P. \& Atabay, B. A Major Outbreak of Severe Acute Respiratory Syndrome in Hong Kong. N. Engl. J. Med. 1 (2003).

99. Guan, Y. et al. Molecular epidemiology of the novel CoV that causes SARS. Lancet 363, 99-104 (2004).

100. Wise, J. Patient with new strain of coronavirus is treated in intensive care at London hospital. BMJ 345, 6455 (2012).

101. Centers, K. Middle East Respiratory Syndrome Coronavirus Outbreak in the Republic of Korea, 2015. Osong Public Heal. Res. Perspect. 6, 269-278 (2015).

102. Lu, X. et al. Real-time reverse transcription-pcr assay panel for middle east respiratory syndrome coronavirus. J. Clin. Microbiol. 52, 67-75 (2014).

103. Huang, P. et al. A rapid and specific assay for the detection of MERS-CoV. Front. Microbiol. 9, 1-9 (2018).

104. Wuhan Municipal Health Commision. Wuhan Municipal Health and Health Commission's Briefing on the Current Pneumonia Epidemic Situation in Our City. http://wjw.wuhan.gov.cn/front/web/showDetail/2019123108989.

105. World Health Organization. WHO Statement Regarding Cluster of Pneumonia Cases in Wuhan, ChinaAvailable online: https://www.who.int/china/news/detail/09-01-2020-who-statement-regardingcluster-of-pneumonia-cases-in-wuhan-china. 
106. Ji, W., Wang, W., Zhao, X., Zai, J. \& Li, X. Homologous recombination within the spike glycoprotein of the newly identified coronavirus 2019-nCoV may boost cross-species transmission from snake to human. (2019). doi:10.1002/jmv.25682

107. Peng Zhou, Xing-Lou Yang, Xian-Guang Wang, Ben Hu, Lei Zhang, Wei Zhang, Hao-Rui Si, Yan Zhu, Bei Li, Chao-Lin Huang, Hui-Dong Chen, Jing Chen, Yun Luo, Hua Guo, Ren-Di Jiang, Mei-Qin Liu, Ying Chen, X.-, Rui Shen, Xi Wang, Xiao-Shuang Zheng, Kai Zhao, Quan-Jiao Chen, F., Deng, Lin-Lin Liu, Bing Yan, Fa-Xian Zhan, Yan-Yi Wang, G.-F. X. \& Shi, Z.-L. Discovery of a novel coronavirus associated with the recent pneumonia outbreak in 2 humans and its potential bat origin. BioRxiv (2020).

108. Labun, K. et al. CHOPCHOP v3: expanding the CRISPR web toolbox beyond genome editing. Nucleic Acids Res. 47, W171-W174 (2019).

109. Ren, X. et al. Enhanced specificity and efficiency of the CRISPR/Cas9 system with optimized sgRNA parameters in Drosophila. Cell Rep. 9, 1151-1162 (2014).

110. Kocak, D. D. et al. Increasing the specificity of CRISPR systems with engineered RNA secondary structures. Nat. Biotechnol. 37, 657 (2019).

111. Liang, M. et al. A CRISPR-Cas12a-derived biosensing platform for the highly sensitive detection of diverse small molecules. Nat. Commun. 10, 1-9 (2019). 